\title{
Le Génie Nb-Sgr "Seigneur du silence" et les dieux qui portent le titre $n b$-sgr dans les lieux saints
}

\section{Dr.salwa kamel ${ }^{\bullet}$}

\section{Conclusions:}

$\mathrm{Nb}$-Sgr "Seigneur du silence" est un génie-gardien du monde infernal, Il joue un rôle tutélaire dans l'Égypte entière, car il était "le maître du silence, qui éloigne le désordre, qui chasse la turbulence d'Égypte (sur lequel on compte pour chasse le secret d'Égypte".

Il garde le soleil comme un être astral, ayant une étoile au-dessus de sa tête dans la douzième heure du jour dans le Livre du jour et de la nuit qui représente la voûte céleste sous la forme d'une déesse dénommée Nout qui recouvrait de son corps le monde habité. Ses jambes étaient placées à l'orient, sa bouche représentait "l'horizon occidental" endroit où disparaissait le soleil couchant.

Les documents qui présentent les génies de Pharbaïthos ont en commun le thème de la résurrection d'un particulier ou d'Osiris:-

- Les cuves de Pharbaïthos et le sarcophage CG. 29305(C $\left.{ }^{1}\right)$

On voit le génie $\mathrm{n}^{0}$ XVIII en forme de momie à tête humaine, coiffée de la couronne blanche et tenant le sceptre $w 3 s$ à deux mains.

- Les représentations des soixante-dix-sept dieux d'Horbeit qui vient d'être signalé à Dendara, c'est-à-dire la figuration symbolique de l'édifice sacré à travers les figures mythiques des génies, il semble que les faucons y aient été déposes pendant 70 jours, ce qui expliquerait le nom égyptien, chambre des 70 . Sur

- assistant professor in the Department of Egyptology faculty of archaeology - Cairo university dr_salwakamel@yahoo.com 
une des parois figurent la grande majorité des 77 génies avec leur nom abrège et sans représentation iconographique. La disposition des cases contenant les noms des divinités de Chedenou évoque d'une manière frappante la répartition des figures sur l'encadrement de la porte du temple de Sokaris de Dendara.

- La présence de certains génies de Pharbaïthos dans la tombe de Pétosiris à Touna el-Gebel, il figure sous forme d'une momie d'Osiris avec un manteau drapé d'où sortent les mains tenant le même sceptre $w 3 s$.

- Le haut des parois de la chapelle de Sokaris à Edfou est décoré de deux petits registres sur lesquels fourmillent les entités protectrices: gardes d'Asebet et gardiens des portes sur la paroi ouest, les 77 de Pharbaïthos sur les parois est et nord, un bandeau définit leur rôle; celui des 77 est, bien sûr, décrit uniquement sur les parois est et nord où ils sont représentés.

- La deuxieme chapelle orientale situe sur le toit du temple d'Hathor, de chaque côté du toit du temple, se trouve un triple ensemble comprenant une cour, une chapelle intermédiaire, et enfin une pièce dans laquelle se déroulaient les actes essentiels des mystères. Il est donc normal que les 77 de Pharbaïthos, dévolus à la garde d'Osiris, y soient représentés.

Le silence était un impératif dans les lieux saints, surtout osiriens; les dieux d'Horbeit, selon les textes-programmes des cuves des taureaux sacrés de Pharbaethos, avaient mission de faire observer cette consigne de silence dans les endroits sous leur surveillance.

L'interdit musical est le plus souvent en relation avec Osiris - ou tout autre dieu mort, il semblait vraisemblable «que ce silence rituel, au voisinage du tombeau d'Osiris, devait s'observer en tout lieu d'Égypte où reposait une relique du dieu défunt, comme aussi sur le territoire de toute butte sainte où étaient ensevelis les dieux initiaux témoins des premiers temps du monde». 
Dans la littérature égyptienne du Moyen Empire, du Paysan éloquent dénonce en effet de manière éclatante l'usage perverti d'un discours d'ordre et de justice par les tenants du pouvoir qui usent de faux-semblants pour couvrir leurs abus, Cet ordre se disparaisse sur un interdit religieux bien attesté en Égypte ancienne, selon lequel il convenait de ne pas élever la voix dans les lieux consacrés aux dieux, et particulièrement à Osiris.

\section{Introduction:}

L'inscription de $n b$-sgr a été comprise qu'un parallélisme étroit existait entre le vocale lui-même et les épithètes le terminant: d'un côté le silence, et d'autre le trouble, le tumulte, donc le bruit ${ }^{1}$.

Le titre $n \boldsymbol{b}$-sgr se compose de:-

L'adjectif $\boldsymbol{n} \boldsymbol{b}$ qui signifie seigneur, a l'implication du propriétaire du possesseur, qui montre la protection d'une divinité dans le temple, les sanctuaires et les villes ainsi que les qualités et les émotions, par exemple la force et la joie ${ }^{2}$. Le verbe sgr qui signifie "pour faire taire", le mot signifie "silence", causal de gr "être silencieux" qui est rarement utilisé à Edfou: dans la Chambre de Sokar, les dieux de la nécropole di "donner silence", où le silence est assimilé à la mort. Dans les rituels de protection, les dieux sont faits au calme avec la commande de Geb que $h r w$ pfy $n$ ce jour-là du silence (le jour du jugement de Horus et Seth $)^{3}$.

\section{Mr.s-gr:}

$\mathrm{Ce}$ nom signifie "elle aime le silence" apparaisse dans l'onomastique dès le Moyen Empire, hors de Thèbes, il n'est pas prouvé qu'il ait désigné une entité divine constituée avant le

\footnotetext{
${ }^{1}$ E.Jelinkova-Reymond, "Les inscriptions de la statue guérisseuse de Djed-Her-le-Sauveur" Bibliothèque d'étude 23 (1956), p.10, nº 1.

H.Goedicke, "Die privaten Rechtsinschriften aus dem Alten Reich", Chr.d'Eg. 90 (1970), 25

${ }^{2}$ Wb II, 227 (5) - 230 (14); WPL, 501.

${ }^{3}$ WbIV, 323-234; WPL, 945-946.
} 
Nouvel Empire ${ }^{4}$. Les manifestations de son culte s'expriment principalement à l'époque ramesside, dans le cadre de la communauté de Deir el-Médineh et des chantiers de celle -ci dans les vallées des Rois et des Reines, à ce titre, elle reçoit des épithètes: dame de l'Occident, la grande déesse de la Nécropole 5 .

郹通 Nb-Sgr "Seigneur du silence" ou "Le Silencieux" est aussi un des génies-gardiens du monde infernal ${ }^{6}$.

$\mathrm{Nb}-\mathrm{Sgr}$ est un génie sous forme de momie du groupe des soixante-dix-sept dieux d'Horbeit, et sa coiffure et ses attributs, il prend la forme d'une statuette gainée, dressée sur un socle rectangulaire et coiffée d'une couronne blanche à uraeus ${ }^{7}$. Son cou est muni d'un collier-menat dont le pendentif retombe dans le dos, et son sceptre, tenu par les mains sortant du maillot, alliant un $w 3 s$ et un ' $n h \underline{h}$ posés sur un $\underline{d} d$. C'est une momie barbue couronnée de l'atef, posée sur un socle rectangulaire de petite taille $^{8}$.

Le corps est enveloppé d'un manteau drapé d'où sortent les mains tenant le même sceptre ${ }^{9}$, donne la couleur verte aux chairs des mains et du visage ${ }^{10}$. Il prend la forme d'un homme criocéphale à Dendera $^{11}$, une momie barbue dressée sur un socle et coiffée ici d'une couronne tni au lieu de l'atef ${ }^{12}$.

\section{Les fonctions de $\mathrm{Nb}-\mathrm{Sgr}$ :-}

-Il joue un rôle tutélaire dans l'Égypte entière, car il était:

${ }^{4} L G G$, III, 343.

${ }^{5}$ D.Valbelle,"Meresger", LÄ IV, 79.

${ }^{6}$ J.CL.Goyon, "Les Dieux-gardiens et la genèse des temples : d'après les textes égyptiens de l'époque gréco-romaine ", Bibliothèque d'étude 93 (1985), 284.

${ }^{7} L G G$, III, 343.

${ }^{8}$ Ibid., 284; LGG, III, 343.

${ }^{9}$ G. Lefebvre, Le Tombeau de Petosiris ; [éd.] Service des Antiquités de l'Égypte,

Bibliothèque générale 29, I, (2007), 153.

${ }^{10}$ Edfou I, pl. XXIX b, gauche (4) = Edfou XI, pl. CCLXXXV, haut (1), 2 à partir de la droite.

${ }^{11}$ Dendera II, pl. LXXXVIII droite, bas (21-23).

${ }^{12}$ Dendera II, pl. LXXXVIII droite, rangée 2, 46-47. 


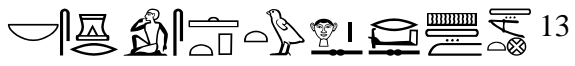

Nb-Sgr-shtp-tw ḥr.s r dr š̌t3 m T3-mry

"Le maître du silence, qui éloigne le désordre, qui chasse la turbulence d'Égypte (sur lequel on compte pour chasse le secret d'Égypte" ${ }^{\prime 14}$.

- Nb-Sgr mentionne dans les textes des Sarcophages comme un génie $^{15}$ qui concerne avec son fonction f vie dans le silence" sans citer l'adjective $n b$.

- Un être astral, garde du soleil, dans la douzième heure du jour ${ }^{16}$ : le quatrième registre, on voit douze divinités ayant toutes une étoile au-dessus de la tête dont les noms sont les suivants: $w d$-nbi et 그용 $n b-s g r^{17}$. (Fig.1 $\left.\mathrm{a}-\mathrm{b}\right)$

Pendant le jour, la barque du dieu Soleil était portée par le fleuve céleste qui coulait sur le corps de la déesse du ciel. Arrivé d'horizon occidental représenté par la bouche de Nout, le soleil cochant était avalé par elle et pénétrait dans les régions mystérieuses de la nuit.

Durant son voyage nocturne, le soleil était halé de l'occident vers l'orient par les étoiles Celles qui ignorent la fatigue et traversait successivement douze régions, "heures" de la nuit, protégées par des portes. Des allusions à cette croyance très ancienne se rencontrent déjà dans les Textes des Pyramides, mais c'est seulement de l'époque du Nouvel Empire que nous sont

${ }^{13} L G G$, III, 343.

${ }^{14}$ P.Vernus, Athribis : textes et documents relatifs à la géographie, aux cultes et à l'histoire d'une ville du delta égyptien à l'époque pharaonique, Bibliothèque d'étude 74 (1978),

$144, \mathrm{n}^{\circ} 18$.

CG 29305, 208.

${ }^{15}$ CT VII, spell 1045.

${ }^{16}$ A. Piankoff, "Le Livre du jour et de la nuit", BdE 13, Le Caire (1942), 26, pl. II.

H.Frankfort, The Cenotaph of Seti l at Abydos, The Egypt Exploration Society Excavation Memoirs 39 II, London 1933, pl. 81.

${ }^{17} L G G$, III 743. 
parvenues des compositions d'ensemble, de vrais livres, qui exposent ces idées ${ }^{18}$.

-Les génies de Pharbaïthos - émanations d'Horus merty - sont chargés à l' origine de protéger Rê, puis, sur l'ordre de ce dernier, de veiller sur la résurrection d'Osiris.

Les documents qui présentent les génies de Pharbaïthos ont en commun le thème de la résurrection d'un particulier ou d'Osiris; ils proviennent aussi bien du Delta que de Moyenne ou de Haute Égypte ${ }^{19}$ :-

1-Les cuves de Pharbaïthos et le sarcophage CG. 29305( $\left.\mathrm{C}^{1}\right)$ :

Liste et procession des dieux-gardien ${ }^{20}$ ont été gravés sur l'extérieur de la cuve rectangulaire en granit gris du second sarcophage d'un $m r m \check{s}^{c}$ et sacerdoce memphite, qui fut, entre autres, prophète d'Osiris, vers le début de la période ptolémaïque $^{21}$. (Fig.2a)

Ce prêtre et officier avait nom $\underline{d} d-h r$; fils du $m r m \check{s}^{e} i \grave{h} m s$, sa mère était la dame $b 3 t$-iy-ti. Sur la paroi extérieure ouest, la bande supérieure est occupée par le bref texte programme relatif aux soixante-dix-sept ${ }^{22}$. On voit le génie $n^{\circ}$ XVIII en forme de momie à tête humaine, coiffée de la couronne blanche et tenant le sceptre $w 3 s$ à deux mains ${ }^{23}$. (Fig.2 b-c)

2-Une paroi d'un petit édifice d'Athribis, la chambre des 70:-

Les représentations des soixante-dix-sept dieux d'Horbeit ${ }^{24}$ qui vient d'être signalé à Dendara, c'est-à-dire la figuration symbolique de l'édifice sacré à travers les figures mythiques des génies, trouvent une bonne illustration dans un ensemble

${ }^{18}$ A. Rusch, Die Entwicklung der Himmelsgüttin Nut zu einer Totengottheit, 1922, p. 13.

${ }^{19}$ S.Cauville, "À propos des 77 génies de Pharbaïthos", BIFAO 90 (1990), p. 115.

${ }^{20}$ Cauville, Ibid., p.116.

${ }^{21}$ CG. 29305, 209, pl.XXI.

${ }^{22}$ Goyon, op.cit., 244, fig. 39.

${ }^{23}$ LGG, III , 742.

${ }^{24}$ Vernus, op.cit., 136. 
d'éléments architecturaux conservés au Musée du Caire, trois panneaux furent taillés sous la $\mathrm{XXX}^{\mathrm{e}}$ (Fig.3a-b), il semble que les faucons y aient été déposes pendant 70 jours, ce qui expliquerait le nom égyptien, chambre des 70 . Sur une des parois figurent la grande majorité des 77 génies avec leur nom abrège et sans représentation iconographique ${ }^{25}$. La disposition des cases contenant les noms des divinités de Chedenou évoque d'une manière frappante la répartition des figures sur l'encadrement de la porte du temple de Sokaris de Dendara ${ }^{26}$.

3-La tombe de Pétosiris à Touna el-Gebel:-

La présence de certains génies de Pharbaïthos ${ }^{27}$ dans la tombe de Pétosiris à Touna el-Gebel ${ }^{28}$, les six premiers génies sont placés au sommet des piliers et les autres repartis sur les piliers A et $\mathrm{B}$ de la tombe; ils figurent deux par deux sur quatre registres qui se lisent de haut en bas, le corps est enveloppé d'un manteau drapé d'où sortent les mains tenant le même sceptre ${ }^{29}$. (Fig.4 a-b)

\section{4-Edfou:-}

Le haut des parois de la chapelle de Sokaris à Edfou est décoré de deux petits registres sur lesquels fourmillent les entités protectrices $^{30}$ : gardes d'Asebet et gardiens des portes sur la paroi ouest, les 77 de Pharbaïthos sur les parois est et nord ${ }^{31}$.

Au-dessus de ces personnages, un bandeau définit leur rôle; celui des 77 est, bien sûr, décrit uniquement sur les parois est et nord où ils sont représentés ${ }^{32}$. (Fig. 5a-b)

${ }^{25}$ Cauville, op.cit.,p. 121.

${ }^{26}$ Goyon, op.cit., 228, fig. 34.

${ }^{27}$ Cauville, op.cit., 122.

${ }^{28}$ Goyon, op.cit., 250.

${ }^{29}$ Lefebvre, Petosiris, II, 84.

${ }^{30}$ Cauville, op.cit., 123.

${ }^{31}$ Goyon, op.cit., 221.

${ }^{32}$ Edfou XI, pl. CCLXXXV, haut (1), 2 à partir de la droite. 


\section{5-La chapelle de Sokaris à Dendera:-}

Par la troupe des petits dieux qui encadrent sa porte, la façade de la chapelle de Sokaris est tout à fait originale. Les trois premières étant séparées par la porte selon la disposition indiquée par le croquis A, il faut commencer par le montant de porte droit, divisé de bas en haut en trois rangées, continuer par l'autre montant, semblablement divisé, et finir par le linteau supérieur reparti en deux rangées $^{33}$, l'ordre ainsi obtenu est celui-là même des premières versions (croquis B) ${ }^{34}$.(Figs. 6-8)

\section{6- La deuxieme chapelle orientale situe sur le toit du temple d'Hathor}

De chaque côté du toit du temple, se trouve un triple ensemble comprenant une cour, une chapelle intermédiaire, et enfin une pièce dans laquelle se déroulaient les actes essentiels des mystères. Il est donc normal que les 77 de Pharbaïthos, dévolus à la garde d'Osiris, y soient représentés.

L'ordre des génies suit, à quelques variations près, celui de l'encadrement extérieur de la chapelle de Sokaris; le croquis suivant indique la manière dont ils sont disposés au deuxième registre $^{35}$. (Fig. 9a-b)

Nb-sgr shpr sgr $m$ Pr.Šnt3yt dsr st $m$ Pr-krs(w)-Wsir- rdi .n.f st.fr $r$

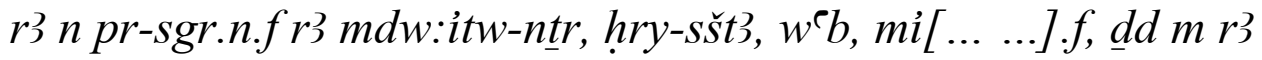

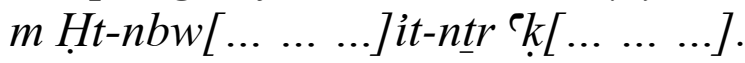

Nebseger: Maître-du-silence, qui impose le silence dans le Sanctuaire-de-Chentayt, dont la place est sacrée dans le Sanctuaire-où-est-enseveli-Osiris(=Dendara)-il prend place à la porte du sanctuaire, il fait taire la bouche qui parle : "Pères divins, prophètes, myste, prêtre, venez [... ...], à la parole

\footnotetext{
${ }^{33}$ Goyon, op.cit., 258.

${ }^{34}$ Cauville, op.cit., 124.

${ }^{35}$ Cauville, op.cit., 127.
} 
assurée dans le Temple d'or [... ... ...]père divin qui entrez $[\ldots . .$. $\ldots]^{36}$.

Le "Maître-du-silence", Nb-sgr, est défini par le texte de Khoiak comme le gardien "accropi sur ses jambes (sndm $h r r d w y . f y)$, une main posée sur la bouche, l'autre sur ses jambes" ${ }^{37}$.

\section{Les dieux qui portent le titre 吸 $n b$-sgr}

\section{- Le dieu Rê}

Le dieu Rê avec son voyage nocturne est le seigneur du silence

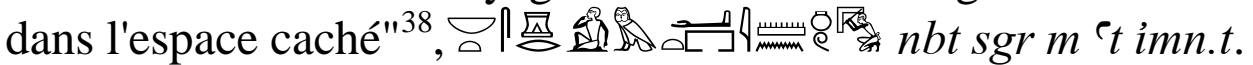

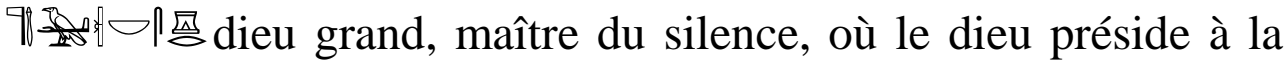
troisième butte $i 3 t$ de la Douat est nommé $n b$-sgr, après quatre lignes de texte, la vignette commence avec deux personnages momiformes à tête humaine, ce sont les grands gardiens de la porte de l'autre monde, derrière eux quatre serpents sortent de leur repaire.

Dans les chapitres 149 et 150 du Livre des Morts, les buttes de l'Au-delà, les quatre régions $i 3 t$ rappellent tout naturellement les quatorze ou quinze qui sont figurées Naville considère les quatre serpents comme représentant les quatre points cardinaux ${ }^{39}$. (Figs. 10-11)

Le défunt veut pouvoir en sortir; il ne veut pas se contenter de la vie de ceux qui ne sont éveillés qu'au court passage du soleil, il veut pouvoir chaque jour, comme le soleil, rentrer sur terre et y courir à son gré, là où les morts ordinaires doivent s'arrêter, lui passe, car il est un auxiliaire indispensable du soleil ${ }^{40}$.

\footnotetext{
${ }^{36}$ Dendara X, 99. pl. 92.

${ }^{37}$ Dendara X, 89, 38-39 Chassinat, Khoaik II, 623.

${ }^{38}$ Urk. VI, 93 (17-18) Louvre 3129.

${ }^{39}$ P.Barguet, Le Livre des Morts, Paris 1967, 214.

${ }^{40}$ G.Nagel,"Un papyrus funéraire de la fin du Nouvel Empire [Louvre 3292 (inv.)]", BIFAO 29 (1929), $75 \mathrm{ff}$.
} 


\section{Le dieu Osiris}

Ce nom $n b-s g r$ attribue à Osiris à cause du nom de l'osireion de Busiris.

C'est à Osiris que revient le titre de $n b-s g r^{41}$, probablement l'iconographie attribuée au génie d'Horbeit soit en accord avec sa nature, l'être momiforme de la troupe de Chedenou offre de trop grandes similtudes avec la représentation d'Osiris. $\mathrm{Nb}$-sgr serait, de la sorte, une incarnation à la fois du pouvoir de faire régener la paix et le calme, dont Osiris était le maitre incontesté, et le silence de l'état des morts ${ }^{42}$.

\section{Le dieu Horus triomphant}

Par ailleurs, quelques dieux ou démons, bons et mauvais, sont liés à cet impératif de l'absence de bruit; ils sont aissi nommés $n b$ $s g r$, parmi eux figurent un Horus triomphant du Delta et ses compagnons $H r n b \quad m 3$ - hrw $n b \quad s g r$ 胳通qui fait triompher Osiris des ennemis car les 997 迢 $n$ tr.w nb.w sgr "dieux seigneurs du silence" le gardent, sur un bloc provenant de la région d'Ousim-Létopolis ${ }^{43}$.

Le naos- $h w t ~ 3 t$ de Tibère et la géographie religieuse du Létopolite.

- L'Abaton du Létopolite. Outre les mentions de hm et shm désignant Létopolis/Ausim, qui permettent d'établir sans le moindre doute la provenance du monument d'Athènes, celui-ci apporte au dossier du second nome du Delta deux mentions toponymiques importantes, relatives à l'Abaton osirien local. Cellesci sont, en outre, mises en relation directe avec des épithètes spécifiques d'Horus définissant la fonction théologique qui lui était dévolue dans ce contexte particulier. La manifestation divine est, en effet, définie comme: $n b m 3^{\circledR}$ hrw et $n b s g r$,

\footnotetext{
${ }^{41}$ Lefebvre, Petosiris I, p.153.

${ }^{42}$ Goyon, Dieux-gardiens, 285.

${ }^{43}$ A.Kamal, "Quelques fragments provenant d'Ousim", ASAE 4 (1903), 93.
} 
épithètes qui ne sont jusqu'alors attestées ainsi associées que sur un bloc de soubassement en granit ${ }^{44}$.

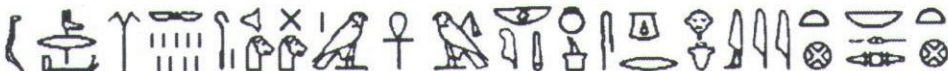

L'élément faisait partie d'une procession géographique mentionnant la personnification féminine $N b$-t-'Iyt de l'Abaton osirien létopolite. Dans le propos attribué à $\mathrm{Nbt-Iyt}$ intervient $\mathrm{Hr}$ $m 3^{\top}$ hrw $n b$ sgr en tant que protecteur d'Osiris à qui il dit: «tu es triomphant, tes ennemis sont tombés et les dieux seigneurs du silence (ntrw nbw sgr) sont ta protection éternellement» ${ }^{45}$.

\section{Les lieux saints où le silence était un impératif:}

Les localités et les noms peuvent également faire l'objet de personnification. Il existait en tout temps de l'époque historique la possibilité de la création d'une telle personnification lors de la rencontre d'une localité particulière en façon humain.

Le silence était un impératif dans les lieux saints, surtout osiriens; les dieux d'Horbeit, selon les textes-programmes des cuves des taureaux sacrés de Pharbaethos, avaient mission de faire observer cette consigne de silence dans les endroits sous leur surveillance ${ }^{46}$.

"Les $b 3 w$ magistraux (wrw) qui sont dans le Khenou-Nekhen, les troupes qui accompagnent Osiris, le grand équipage du Domainedu-dieu, qui font régner le silence dans Areq-Heh, qui écoutent le décret divin émis dans la Douat, qui ne cessent d'accomplir ce qu'il dit dans leur nome" ${ }^{47}$.

44 Retrouvé dans les années 1900, remployé dans la mosquée du village de Kafr Sidi Moussa appartenant au site d'Ausim.

${ }^{45}$ V.I. Chrysikopoulos - J.Goyon, "Un témoin inédit des auvres pieuses de l'empereur Tibère à Létopolis de Basse-Égypte", CdE 84 (2009), 131.

${ }^{46}$ A.Gutbub, "Textes fondamentaux de la théologie de Kom Ombo", Bibliothèque d'Étude ; 47, 1973, p. 172ff.

${ }^{47}$ Goyon, op.cit., 220. 
L'interdit musical est le plus souvent en relation avec Osiris ${ }^{48}$ ou tout autre dieu mort, il semblait vraisemblable «que ce silence rituel, au voisinage du tombeau d'Osiris ${ }^{49}$, devait s'observer en tout lieu d'Égypte où reposait une relique du dieu défunt, comme aussi sur le territoire de toute butte sainte où étaient ensevelis les dieux initiaux témoins des premiers temps du monde» 50. di sgr; selon ce texte, ce seraient donc les 77 d'Horbeit qui auraient eu mission de faire respecter l'interdit des Abatons osiriens musique et bruit, le dieu étant, par excellence, le $n b s g r$ "seigneur du silence"

L'une des invalidations les plus pressantes consistait à chasser de ce lieu sacré toute musique ou tout bruit qui pût évoquer quelque cérémonie joyeuse ou quelque partie de plaisir: le corps du "dieu qui aime le silence" devait reposer en toute qui étude, loin de la trouble qui était l'indication de la vie, sans que rien d'extérieur ne vînt troubler la calme de son tombeau ${ }^{52}$.

Il a également montré pour quelle raison la trompette ${ }^{53}$ était prohibée à Busiris, mais il ne s'agit que d'un seul des instruments de musique incriminés.

\footnotetext{
${ }^{48}$ S.Emerit, "À propos de l'origine des interdits musicaux dans l'Égypte ancienne", BIFAO 102 (2002), p. 189-210.

${ }^{49}$ S. Sauneron, Esna III, Le Caire, 1968, p. 14, n 197, 24 -26.

${ }^{50}$ H.Junker, "Das Götterdekret über das Abaton", (DAW, Wien 56, 1913), p. 21-22.

${ }^{51}$ Dendara II, 158 (10) ; E.Chassinat, Les mystères d'Osiris au mois de Khö̈ak, II (1968), p. $623 \mathrm{ff}$.

52 S.Sauneron, "L'Abaton de la champagne d'Esna", MDAIK 16 (1958), 171.

${ }^{53}$ S.Sauneron,"Remarques de philologie et d'étymologie (\$ 36-45) ", BIFAO 64 (1966), $12-$ $4 \S 38$.
} 
- Décret divin de l'Abaton de Philae ${ }^{54}$.

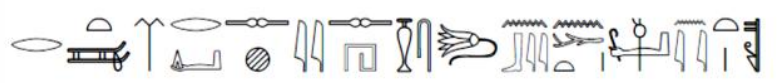

Il est interdit de battre du tambour-sh(r) et de chanter-hs accompagné de la harpe-bynt ou de la flûte-wdnyt (r tm rdi shi $\operatorname{sh}(r)$ hsi $\operatorname{n}$ bynt $w$ dnyt $)^{55}$.

- Le deuxième texte est inscrit sur une colonne de la salle hypostyle du temple d'Esna:

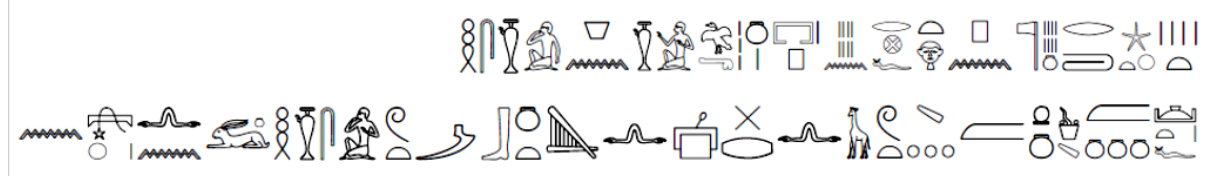

"Faire la grande offrande de toute bonne chose, chanter par les chanteurs masculins de ce temple; face à ce dieu, jusqu'à ce que la $4^{o}$ heure de la nuit soit en cours. Mais ne pas chanter en s'accompagnant sur la harpe, ni battre le tambour, ni lancer de sonneries de trompettes dans l'enceinte de sa butte" ${ }^{156}$.

- Kôm Ombo 597, 5-6 ${ }^{57}$ :

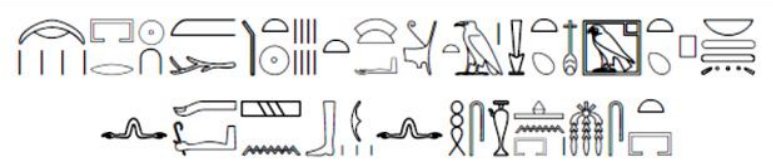

Le troisième texte relatif à un interdit musical est issu de l'un des calendriers des fêtes du temple double de Kôm Ombo. Dédié à Sobek-Re et consacré aux fêtes de la nécropole et du mammisi, il

${ }^{54}$ J. Yoyotte, P. C.Harvet, S. Gompertz, Strabon. Le voyage en Égypte, Paris 1997, p. 260 261.

${ }^{55}$ H.Junker, Abaton, 21-22.

${ }^{56}$ S. Sauneron, Esna III, Le Caire, 1968, p. 14, no 197, 24 -26 ; H.Junker, Abaton 25-26.

${ }^{57} \mathrm{~J}$. DE Morgan, Catalogue des monuments et inscriptions de l'Égypte antique, $1^{\text {ère }}$ série, t. 3, Kôm Ombos II, Vienne, 1909, p. 53; Fr. Daumas, Mammisis, p. 240-241; A. Gutbub, Kôm Ombo, p. 335, n. m); A. Grimm, Festkalender, p. 92-95; S. El-Sabban, Temple Festival Calendars of Ancient Egypt, Trowbridge, 2000, p. 157, pl. 33. 
est inscrit sur le soubassement de la partie droite de la salle des «apparitions», paroi oust.

Quatrième mois de la saison peret, jour 10 : après la huitième heure,sortie en procession de Tasenetneferet-Hathor et de Panebtaoui. Ne pas saisir la trompette- $n b$. Ne pas chanter-hs $(t)$.

Cette description semble correspondre à $H r y-m{ }^{`}{ }^{`} t$ le dieu de tombes de la vallée des reines a-t-il subi une mutation pour devenir le maître du silence.

$N b$-sgr, lieu où le silence était depuis toujours recommandé ${ }^{58}$.

Les deux génies $N b$-sgr et $H r y-m{ }^{`} t$ sont deux protecteurs d'Osiris dans son tombeau ${ }^{59}$.

Sur la stèle Chicago OI $8798^{60}$,

Le Grand Prêtre d'Amun dans Djeser-Djeseru, Senenu; il dit: Si l'on se transforme [Parce que jusqu'à la justesse et le silence, mon âme est appelée à partager la nourriture dans le domaine] des Roseaux ${ }^{61}$. (Fig.12)

\section{Dans la littérature égyptienne du Moyen Empire:-}

L'enseignement pour mrj $k 3 R^{r}$ fournit certainement l'une des évocations les plus explicites du rôle de la rhétorique dans la vie politique en Égypte ancienne ${ }^{62}$.

Au Moyen Empire, le Conte de l'Oasien (ou du Paysan éloquent) dénonce en effet de manière éclatante l'usage perverti d'un discours d'ordre et de justice par les tenants du pouvoir qui usent

${ }^{58}$ A.Gutbub,Théologie de Kom Ombo I, 172.

${ }^{59}$ Urk. VI, 93, 17 et 99, 20.

${ }^{60}$ LGG, III, 743.

${ }^{61}$ E.Brovarski, "Senenu,High Priest of Amun at Deir El-Bahari", JEA 62 (1976), p. 59, pl.11.

${ }^{62} \mathrm{Ph}$. Derchain, «Éloquence et politique. L'opinion d'Akhtoy», RdE 40, 1989, p. 37 -47; édition du texte: J.Fr. Quack, Studien zur Lehre für Merikarem, GOFIV/23, Wiesbaden, 1992. 
de faux-semblants pour couvrir leurs abus ${ }^{63}$. Dès le début du récit, cette pratique est mise en évidence à travers le personnage de $n m$ - $t j n h t$. Alors qu'il vient de déposséder le paysan de son bien $^{64}$, il fait taire ses pleurées en disant ${ }^{65}$ :

$m$ k hrw.k shtj

N'élève pas la voix, paysan!

mk tw $r$ dmj $n$ nb sgr

Vois, tu approches du domaine du Seigneur du Silence!

Cet ordre se disparaisse sur un interdit religieux bien attesté en Égypte ancienne, selon lequel il convenait de ne pas élever la voix dans les lieux consacrés aux dieux, et particulièrement à Osiris ${ }^{66}$. Cet interdit fait l'objet ici d'un détournement délibéré, car $n m-t j n h t$ en fait un argument pour réduire au silence celui qu'il a spolié. Cet épisode initial est emblématique à plusieurs titres. En premier lieu, l'allusion au «Seigneur du silence» annonce la confrontation du paysan avec le grand intendant le juge $r n . s j{ }^{67}$.

${ }^{63}$ Papyrus Chester Beatty IV, v 2,5 - 3,11 (trad. dans P. Grandet, Contes de l’Égypte ancienne, Paris,1998, p. 161-162 et p.187), et les analyses de J. Assmann, «Gibt es eine «Klassik» in der ägyptischen Literaturgeschichte? Ein Beitrag zur Geistesgeschichte der Ramessidenzeit», ZDMG Suppl.6, 1985, p. 35 -52.

${ }^{64}$ R.B. Parkinson, «Teachings, Discourses and Tales from the Middle Kingdom», in S. Quirke (éd.), Middle Kingdom Studies, New Malden, 1991, p. 105-122.

${ }^{65}$ [Oasien B1 57-58].

${ }^{66}$ A. Gutbub, Textes fondamentaux de la théologie de Kom Ombo, BdE 47, Le Caire (1973), p. 172-174. P.J. Frandsen, «On the Avoidance of Certain Forms of Loud Voices and Access to the Sacred», in W. Clarysse, Egyptian Religion.The Last Thousand Years. Studies Quaegebeur, OLA 85 Louvain, 1998, p. 975-1000. Voir aussi H.De Meuleunaere, in O. Firchow, Ägyptologische Studien. Fs. Grapow, Berlin, 1955, p. 228-229; Y. Koenig, Le papyrus Boulaq 6, BdE 87, Le Caire, 1981, p. 102-103, n. (i); J.-Cl. Goyon, op.cit, 93; Cf. P. Vernus, op.cit, p. 40 et n. $18-19$.

${ }^{67}$ L.Coulon, "La rhétorique et ses fictions. Pouvoirs et duplicité du discours à travers la littérature égyptienne du Moyen et du Nouvel Empire", BIFAO 99 (1999), p. 103,105. 


\section{Planches}

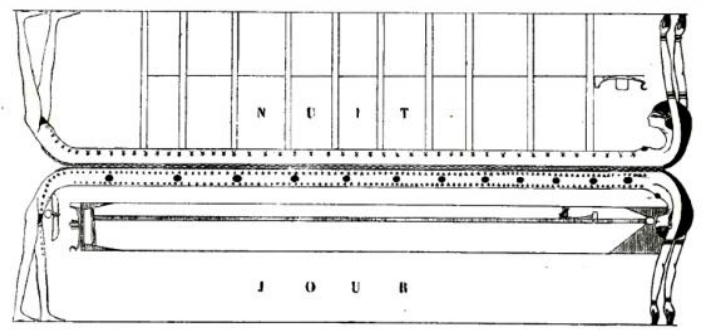

Fig.1 a

Le Livre du jour et de la Nuit dans la salle du Sarcophage de Ramsés VI.

A. Piankoff, "Le Livre du jour et de la nuit", p.II.

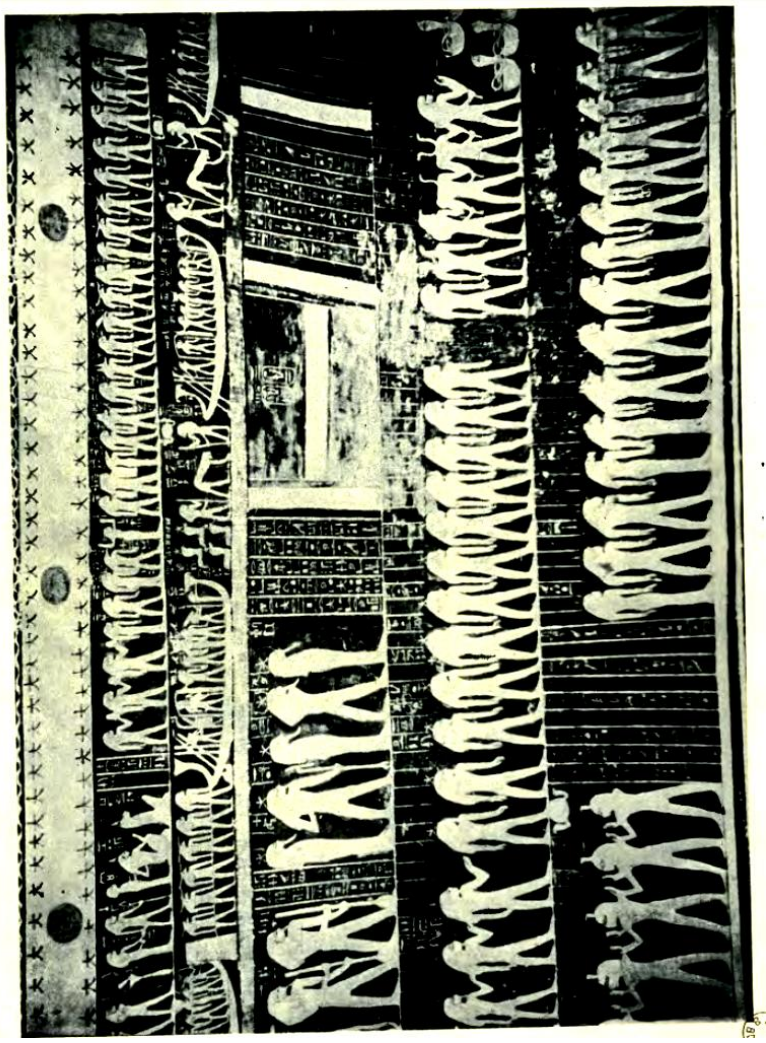

Fig.1b.

A. Piankoff, Ibid., pl. II. 

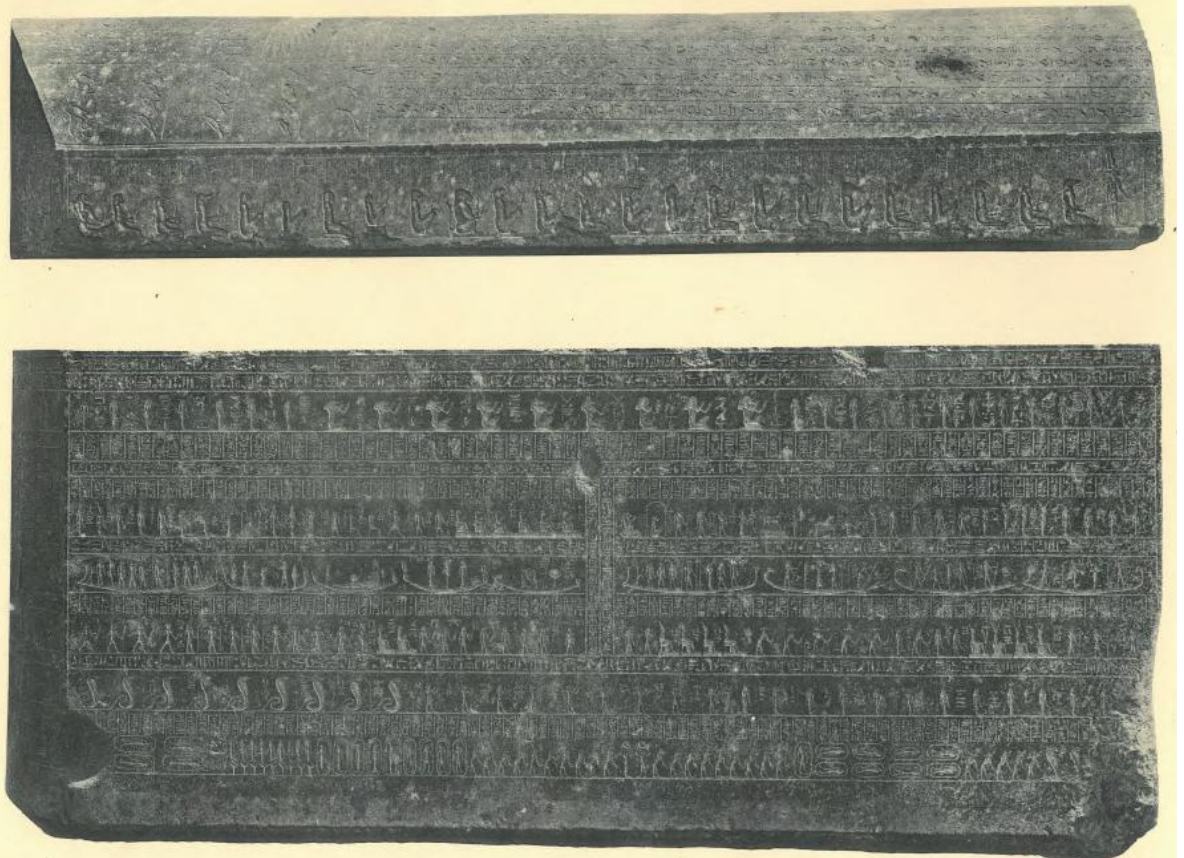

Fig.2a

Maspero, CG. 29305, pl.XXI.

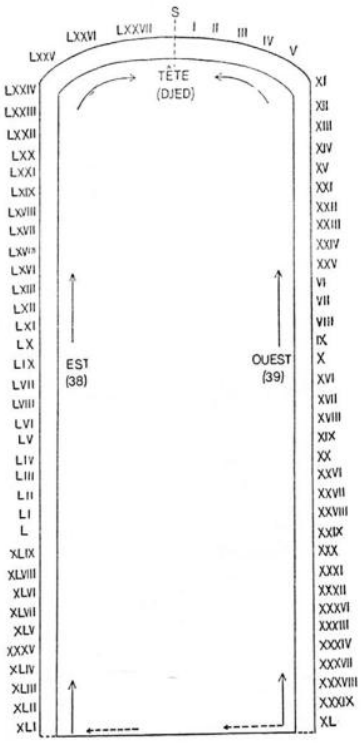

Fig.2b

Goyon, Dieux-Gardiens, 244, fig. 39. 


\begin{tabular}{lllllllll}
\hline Désignation & Ordre $^{\circ}$ & PH. 86718 & PH. 86717 & CG. 29305 & ATHRIBIS & EDFOU & D. 1 & D. 2 \\
\hline$N b S g r$ & 18 & III & - & - & - & - & - & - \\
\hline
\end{tabular}

Fig. 2 c

Tableau pour indiquer l'ordre de génie $\mathrm{Nb}-\mathrm{Sgr}$

Sur le sarcophage $C G .29305\left(C^{1}\right)$

d'après S.Cauville.

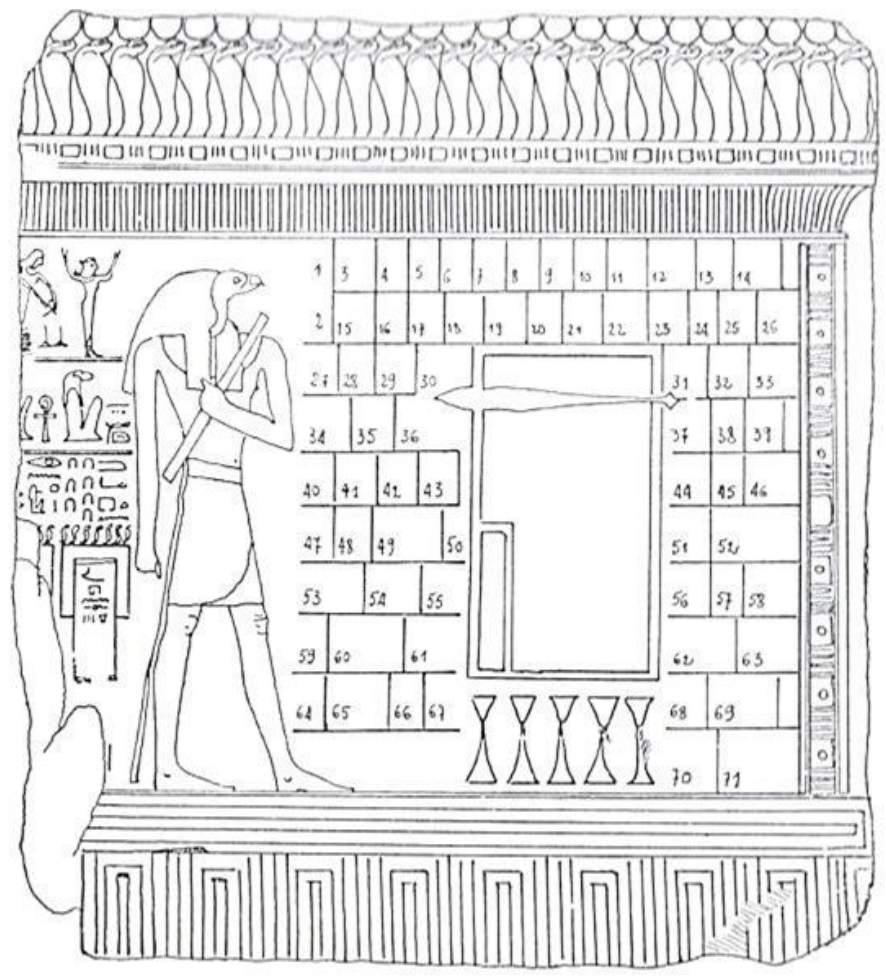

Fig.3a

Une paroi d'un petit édifice d'Athribise $\mathrm{Nb}-\mathrm{Sgr}$ .la chambre des 70 .

Goyon, Dieux-Gardiens., 228, fig. 34. 


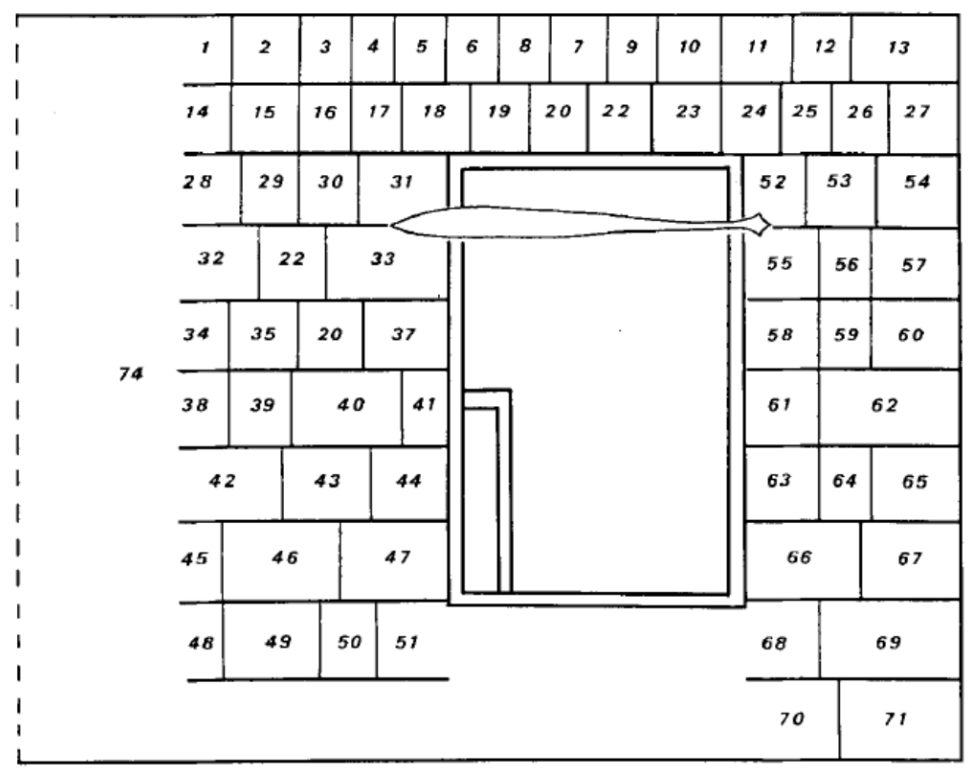

Fig.3b

Tableau pour indiquer l'ordre de génie $\mathrm{Nb}-\mathrm{Sgr}$

la chambre des 70 .

d'après Cauville, 77 génies de Pharbaïthos, p.121.

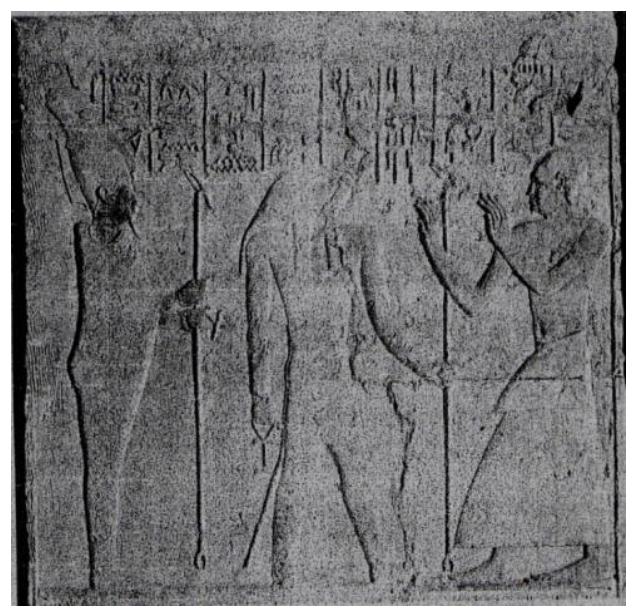

Fig.4a

La tombe de Pétosiris à Touna el-Gebel

Lefebvre, Petosiris, II, 84. 

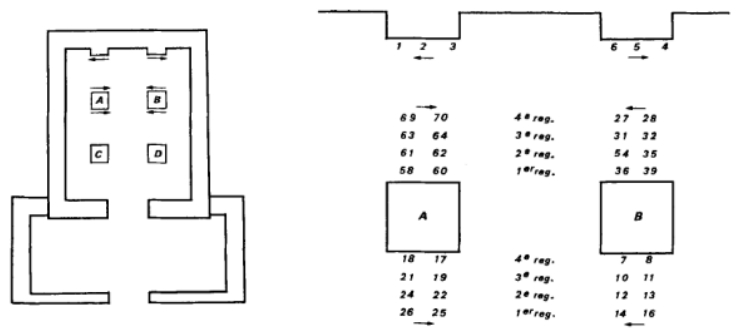

Fig.4b

Plan et tableau pour indiquer la position de génie $\mathrm{Nb}-\mathrm{Sg} r$

Cauville, op.cit., 122.

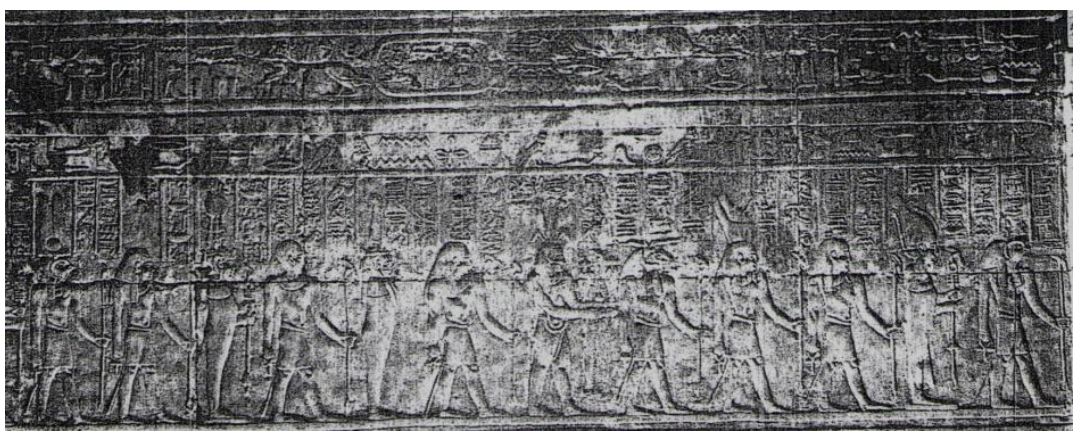

Fig. 5a

Edfou XI, pl. CCLXXXV, haut (1), $2^{\mathrm{e}}$ à partir de la droite.

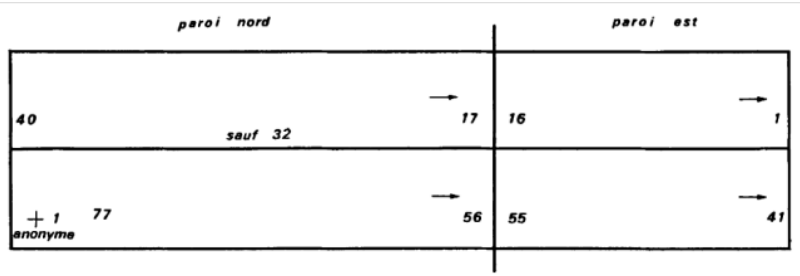

Fig. 5b

Tableau pour indiquer la position de génie $\mathrm{Nb}-\mathrm{Sgr}$

Cauville, op.cit., 123. 


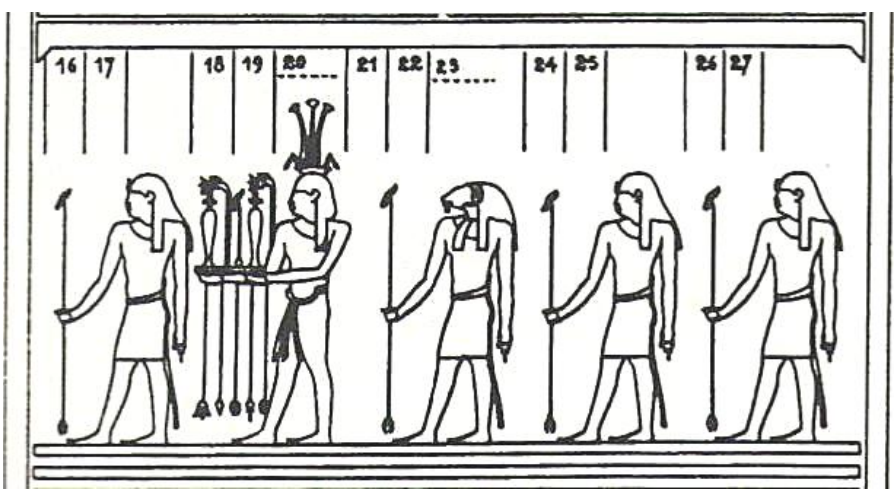

Fig.6

Dendera II, pl. LXXXVIII droite, bas (21-23).

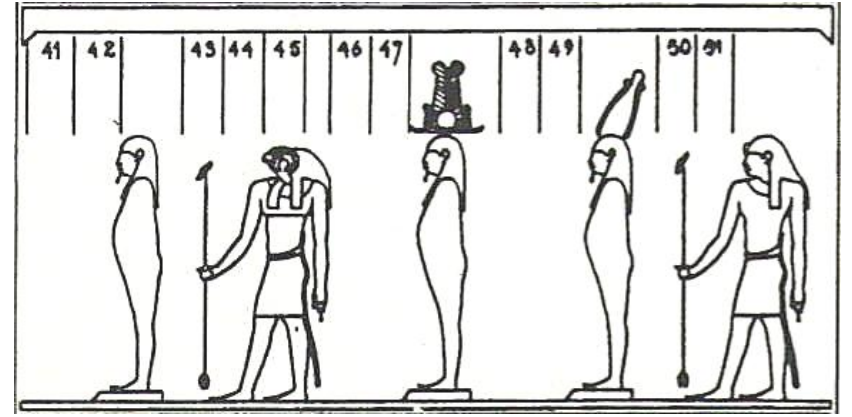

Fig.7

Dendera II, pl. LXXXVIII droite, rangée 2, 46-47.

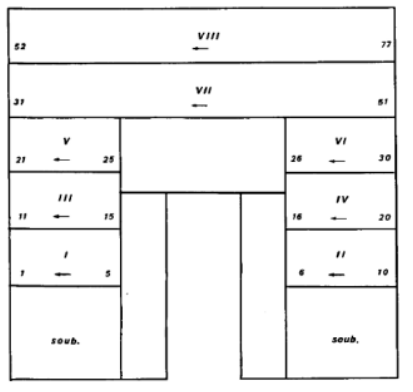

Croquis A.

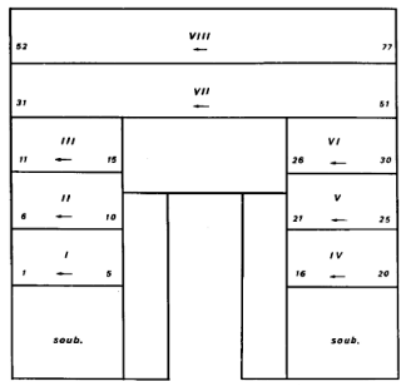

Croquis B.

Fig.8

Tableau pour indiquer la position de génie $\mathrm{Nb}-\mathrm{Sgr}$

Cauville, op.cit., 124. 


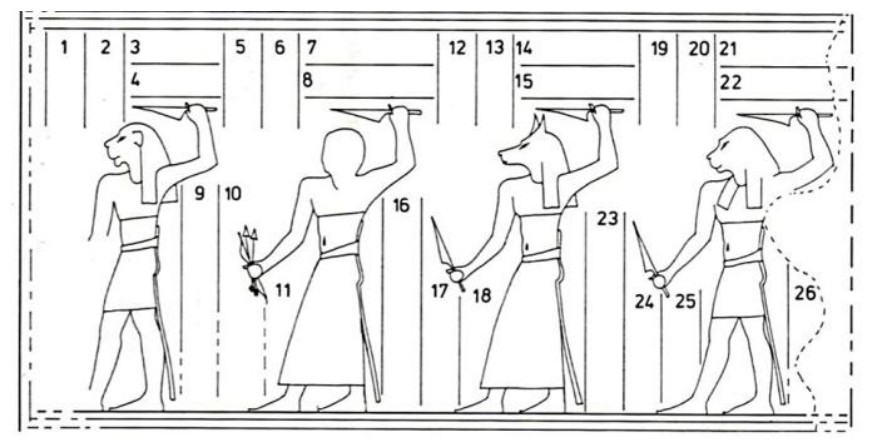

Fig.9a

Dendara X, 89

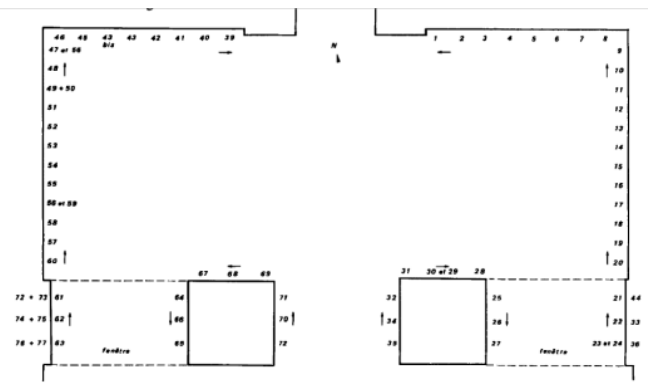

Fig.9b

Tableau pour indiquer la position de génie $\mathrm{Nb}-\mathrm{Sgr}$

Cauville, op.cit., 126.
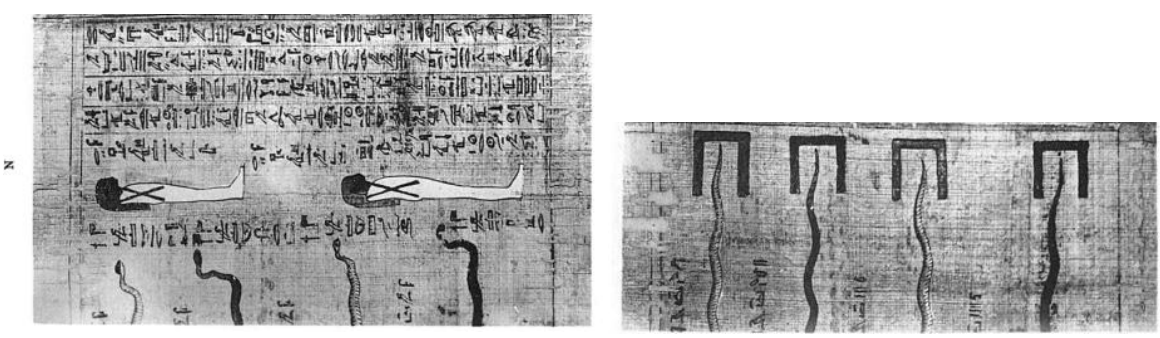

Fig.10

G.Nagel,"Un papyrus funéraire [Louvre 3292 (inv.)], pl.V-VI. 


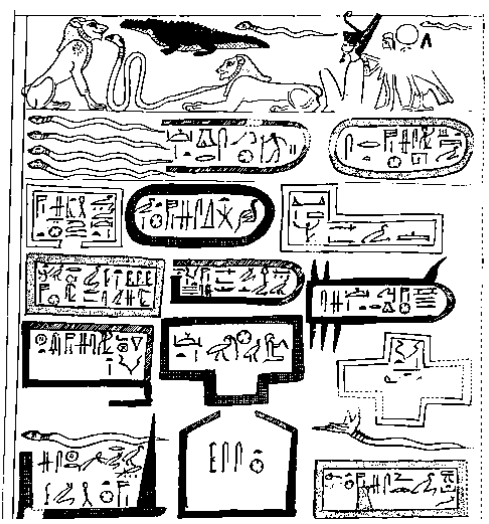

Fig. 11

Chapitres 149-150.

P.Barguet, Le Livre des Morts, 214.

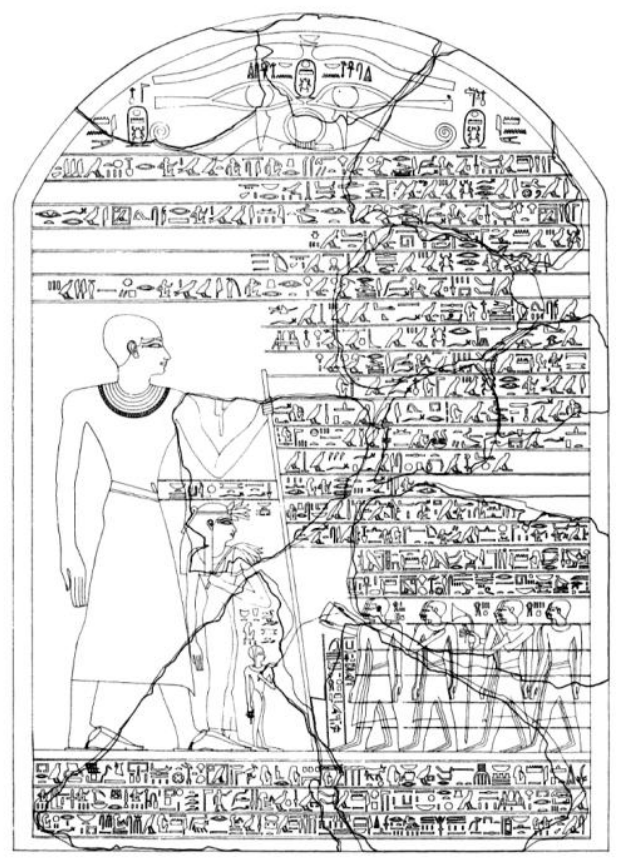

Fig.12

E.Brovarski, "Senenu,High Priest of Amu", pl.11A. 


\section{الحامهى (المعصود) نـب - سمر}

" سبد السكون والمعبودات التى حملت لقب نب - سجر فى الاماكن المقدسة " د.سلوى احمد كامل

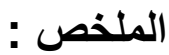

يعتبر "نب - سجر" و احد من الحراس الحامبين فى العالم السفلى، كان لله دورَ حارساً فى مصر كلها لأنه كان "سيد الصمت أو السكون ويطلق عليه الصامت أو أون الساكن ، و الذى يبعد الفوضى، و يدفع الاضطر اب بعيداً عنها.

من وظائفه إنه كان بحرس الثمس ضمن مجموعة تتكون من اثنى عشر حارساً في الساعة الثانية عشرة في كتاب النهار و اللبل، يوجد العديد من الوثائق التى تمنثل

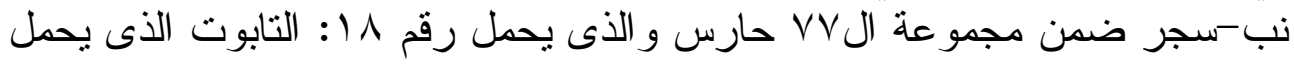
رقم 29305 CG و حجرة السبعين فى اتريب وفى مقبرة بتوزوريس فى تونة الجبل ومقصورة سوكر فى معبد ادفو و أيضاً فى المقاصير الاوزيرية بمعبد دندرة. كان الصمت بالأمر فى الأماكن المقدسة وخاصة الأوزيرية، وتحريم الموسبقى أيضاً وكذلك العزف على الطبلة و الهارب و الناى فى الاماكن التى بها أى بقايا مدفونة للجسد الأوزيرى.

في الأدب المصري من عصر الدولة الوسطى، يدين "الفلاح الفصبح" الو اقع بشكل و اضح من أجل التعبير عن العدالة، يظهر هذا الأمر بالمنع المؤكد فى مصر القديمة بعدم رفع الصوت فى الأماكن المكرسة للمعبودات وخاصة أوزيريس. 